\title{
Erratum: Dynamical manifestation of an evolving Berry phase as a frequency shift of the resonance transition between two eigenstates [Phys. Rev. A 84, 062103 (2011)]
}

\author{
Koichi Toriyama, Akihide Oguchi, and Atsuo Morinaga \\ (Received 5 May 2016; published 23 May 2016)
}

DOI: 10.1103/PhysRevA.93.059903

In Fig. 4, the frequency shift in the vertical axis is the shift of the applied rf frequency, not the shift between the two states, as the reason stated in the erratum [1]. In the right-handed rotation, the frequency shift between the $m_{F}=-1 \rightarrow m_{F}=1$ states should be increased by $2 f$ for $0 \leqslant \theta \leqslant \pi / 2$ and decreased by $2 f$ for $\pi / 2 \leqslant \theta \leqslant \pi$, while the frequency shift between the $m_{F}=1 \rightarrow m_{F}=-1$ states is vice versa. Then the frequency shift is equal to the time derivative of the Berry phase $-4 \pi(1-\cos \theta)$ for a whole rotation. Thus we confirmed that the Berry phase depends on the spin component and the signed solid angle, but does not depend on the sign and the magnitude of the $g$ factor, as originally predicted by Berry [2]. Therefore, the Abstract and the Conclusion should be corrected similarly.

[1] A. Morinaga and Y. Hasegawa, Phys. Rev. A 93, 059902 (2016).

[2] M. V. Berry, Proc. R. Soc. London, Ser. A 392, 45 (1984). 